\title{
A MULTIBODY DYNAMIC MODEL FOR EVALUATING THE VIBRATING MODES OF GEAR TRAIN SYSTEMS
}

\author{
R. P. MONTEIRO, R. VIDONI \& F. CONCLI \\ Faculty of Science and Technology, Free University of Bozen-Bolzano, Italy
}

\begin{abstract}
Nowadays, the use of gearboxes in the mechanical sector has significantly increased due to the various possible applications and new materials available on the market. Moving through each application, from robotics to the automotive sector, the use of gearboxes ensures high efficiency with a compact structure. Generally, gearboxes are composed by gears, shafts and bearings, and each of these components can be subjected to failures. Therefore, in the analysis of each mechanical component it is central to let the system operate properly. In fact, the presence of a damage can lead to a slight variation of system properties (e.g. stiffness). To design even more reliable gearboxes, it results fundamental to monitor the system's health state and the damage progress. To better understand these phenomena, a numerical study is here presented. A back-to-back gear rig (fixed-axis two-stage test rig) is used as reference. It was developed an effective multibody dynamic model that exploits a combination of two different approaches - the Lumped Parameter Method (LPM) and the Finite Element Method (FEM). In this work, the effect of different operative and loading conditions was studied. In particular, the effect of damages on the eigenfrequencies and on the vibrational spectra was investigated based on numerical simulations. The stiffnesses of the gear train components, used in the LPM, were estimated by means of dedicated FEM simulations. The results of the LPM were validated with experimental data acquired on a real healthy back-to-back rig. Moreover, the effect of a tooth root damage on the vibrational spectra was analysed. The stiffnesses of the system's components affect considerably the eigenfrequencies. As predicted by the FEM simulations, in presence of damage, the stiffness of the teeth varies significantly, affecting the vibrational spectra. Therefore, this work can be an effective starting point to setup a monitoring strategy of gearboxes.

Keywords: dynamic modelling, finite element method (FEM), gear trains, gearboxes, gears, lumped parameter method (LPM), multibody modelling (MBM), multibody systems.
\end{abstract}

\section{INTRODUCTION}

Generally, gearboxes are used to transmit mechanical torque from a system to another. They are composed by at least two gears which have usually different dimensions. In addition, gearboxes are characterized by high efficiencies and their performances are fundamental in the transmission of mechanical energy due to their versatility and applicability to any rotating system. However, these mechanical solutions are subjected to intense working conditions, which may cause failures and damages within the system.

The multibody modelling (MBM) approach represents a powerful tool to investigate the dynamic of a geared system. It can be an effective tool to study/optimize the vibrational behaviour of a system [1] relying on lumped parameters [2]. Moreover, bodies can be considered flexible and/or rigid. This type of analysis is based on physical models that describe the characteristic vibrations in terms of amplitude, frequency and phase modulation, providing important results that can help to better understand the failure modes [3].

Vibration can be referred to as a physical movement or motion of a rotating machine. To measure it, the frequency and amplitude must be converted into electric signals by means of a transducer. An acceleration measure shows how fast (frequency) and how much (amplitude) the analysed system vibrates. While a frequency variation is a key effect to identify the type of the problem, the amplitude shows the relative severity of the damage [4]. In addition, vibrations generate oscillatory motions that produce forces that can affect the systems behaviour [5]. 
A gearbox system is made of interacting bodies such as gears, bearings, shafts and housing (supporting structure). Gears rotate around fixed (or moving) axes transmitting the power via meshing teeth [6]. Generally, a gear train can be modelled as a flexible multibody system that, owing to the interaction of deformable bodies [7], leads to the identification of the system response.

The MBM can be created through the LPM where components are schematized with concentrated masses and inertias, and the interactions between bodies are modelled via springs and dampers. Masses and inertias can be estimated by means of Computer Aided Design (CAD) software. For the evaluation of elements' stiffnesses, analytical formulations and/or numerical approaches should be employed. Indeed, for components having complex geometries such as the ones of a gearbox (e.g. gears, shafts and bearings), numerical approaches - such FEM - are required. Such a LPM is helpful to identify the eigenfrequencies of the system. Any variation in the lumped parameters (for example, related to the nucleation of cracks) reflects directly on the frequency spectra. This property can be exploited to identify possible problems that occur in a mechanical system during operation.

\section{DYNAMIC MODEL}

To validate the results of numerical simulations, a back-to-back rig was tested experimentally (Fig. 1). The rig is composed by 2 shafts, 12 bearings and two gear pairs (test and slave gearboxes). Each gear pair has the same centre distance and the same gear ratio. The gears are connected to each other via compliant shafts (shafts 1 and 2). The shaft 2 is composed by a servo-hydraulic torque actuator (coupler) that decouples the two semi shafts (positive shaft 2 and negative shaft 2). This mechanism, arranged in a closed mechanical loop, applies a rotation to the two semi-shafts to induce equals and opposite torques in the tested gears. This configuration of the system can be used to test gearing at varying speed.

The mechanical response of the real system was reproduced numerically [8]. In this regard, a combination of a LPM and a FEM was used to describe the test-rig with a lean model. This

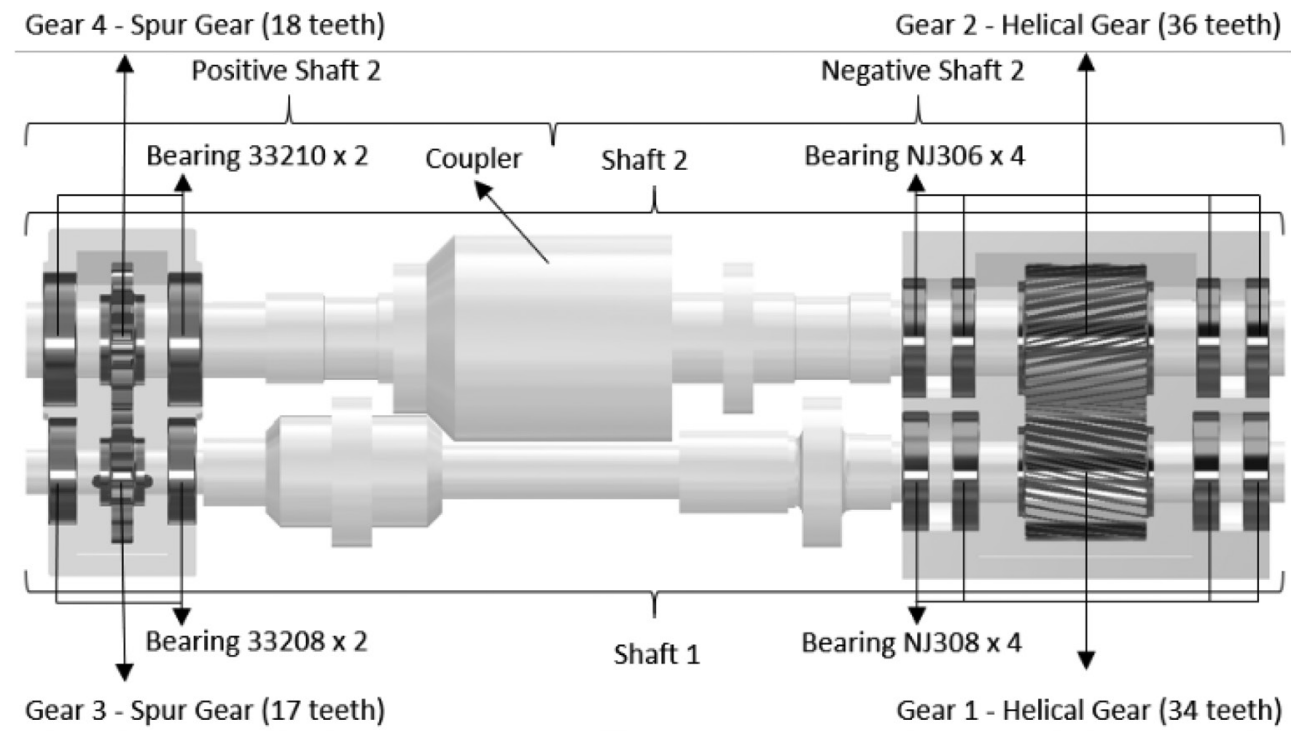

Figure 1: Gear train system. 
combined approach can simulate the effect of different operative conditions considering the eigenfrequencies of the system. Equivalent masses and inertias were calculated knowing the size and material of gearboxes components while the stiffnesses were determined through the FEM simulations.

\subsection{Lumped parameter method (LPM)}

The lumped parameter method approximates the real system exploiting discrete entities that are close to the real behaviour of the analysed component. The LPM method is based on lumped masses and inertias concentrated in specific points [9-10]. In this research, damping was neglected, since the objective of this paper was to determine the eigenfrequencies of the system.

The physics-based model provides a framework based upon physical laws. It describes the behaviour of the system using the principles of mass, momentum, energy conservation, and Newton's laws of motion. The model can be described with the following equation:

$$
m \ddot{x}(t)+c \dot{x}(t)+k x(t)=F(t)
$$

where the system parameters are mass $m$, damping $c$ and stiffness $k$; the system responses are acceleration $\ddot{x}$, velocity $\dot{x}$ and position $x$, while $F(t)$ is the time-dependent external force. Equation (1) is recognizable as the equation of motion for a damped and forced system with a single DOF. Having the analysed system $n$ DOF, equation (1) should be rewritten in matrixial form:

$$
[M]\{\ddot{x}(t)\}+[C]\{\dot{x}(t)\}+[K]\{x(t)\}=\{F(t)\},
$$

where $M$ is the mass matrix, $C$ is the damping matrix and $K$ is the stiffness matrix that represent the state variables for a given system. $F(t)$ is the forcing function providing input to the system, and $x(t)$ is the overall displacement vector that represents the output of the model. Assuming no forces and no damping equation, (2) is reduced to:

$$
[M]\{\ddot{x}(t)\}+[K]\{x(t)\}=0 .
$$

The corresponding eigenvalue problem of the undamped free system is numerically solved through the following equation (4) in order to find the undamped eigenfrequencies $\omega_{\lambda}$ and the corresponding mode shapes $Q_{\lambda}\left(\lambda \in\left[1, N_{d o f}\right]\right)$ :

$$
K Q=\omega^{2} M Q
$$

\subsubsection{Vibration differential equation of the gear train system}

Figure 2 represents the virtual model of the fixed-axis two-stage back-to-back test rig. The system was modelled with 3 DOF on each gear, assuming translation along $x$ and $y$ axis as well as rotation around the $z$ axis. Stage 1 consists of gears 1 and 2 (helical gear pair), while stage 2 consists of gears 3 and 4 (spur gear pair). These assumptions lead to a model having 12 DOF. Gears were reduced to lumped masses/inertias. The mating teeth and the bearing reduced to linear springs. The two shafts were modelled as torsional springs. Additionally, 
the input speed of the gear train was set to $3,000 \mathrm{r} / \mathrm{min}$, the maximum power transmitted to $314.16 \mathrm{~W}$, and the resistant torque resulted therefore $1 \mathrm{Nm}$

The gear train parameters of the system are shown in Table 1. The LPM is shown in Fig. 2, where $k_{i}, k_{j}, k_{m}, k_{n}$ are the stiffnesses of the bearings, and $k_{i} x_{1}, k_{j} x_{2}, k_{m} x_{3}, k_{n} x_{4}, k_{i} y_{1}$, $k_{j} y_{2}, k_{m} y_{3}, k_{n} y_{4}$ are the forces of the bearings in the $x$ and $y$ directions $(i=1-4, j=5-8$,

Table 1: Gear train parameters.

\begin{tabular}{lllllll}
\hline Parameters & Symbol & Stage 1 & \multicolumn{3}{c}{ Stage 2 } & Units \\
\cline { 3 - 6 } & & $\begin{array}{l}\text { Helical } \\
\text { gear 1 }\end{array}$ & $\begin{array}{l}\text { Helical } \\
\text { gear 2 }\end{array}$ & $\begin{array}{l}\text { Spur } \\
\text { gear 1 }\end{array}$ & $\begin{array}{l}\text { Spur I } \\
\text { gear 2 }\end{array}$ & \\
\hline No. of teeth & $N$ & 34 & 36 & 17 & 18 & - \\
Module & $M$ & 2.5 & 2.5 & 5.0 & 5.0 & - \\
Mass & $m$ & 3.1 & 3.3 & 3.1 & 3.3 & $\mathrm{Kg}$ \\
Moment of inertia & $J$ & 0.0122 & 0.0146 & 0.0122 & 0.0146 & $\mathrm{Kg} \mathrm{m}{ }^{2}$ \\
Pitch circle diameter & $d$ & 0.0888 & 0.0941 & 0.0888 & 0.0941 & $\mathrm{~m}$ \\
Pressure angle & $\gamma$ & 20 & 20 & 20 & 20 & $\mathrm{deg}$ \\
Helix angle & $\beta$ & 12 & 12 & 0 & 0 & $\mathrm{deg}$ \\
Contact ratio & $\varepsilon$ & 1.0588 & 1.0588 & 1.0588 & 1.0588 & - \\
Young's modulus & $E$ & 200 & 200 & 200 & 200 & $\mathrm{GPa}$ \\
Poisson's ratio & $v$ & 0.3 & 0.3 & 0.3 & 0.3 & - \\
\hline
\end{tabular}

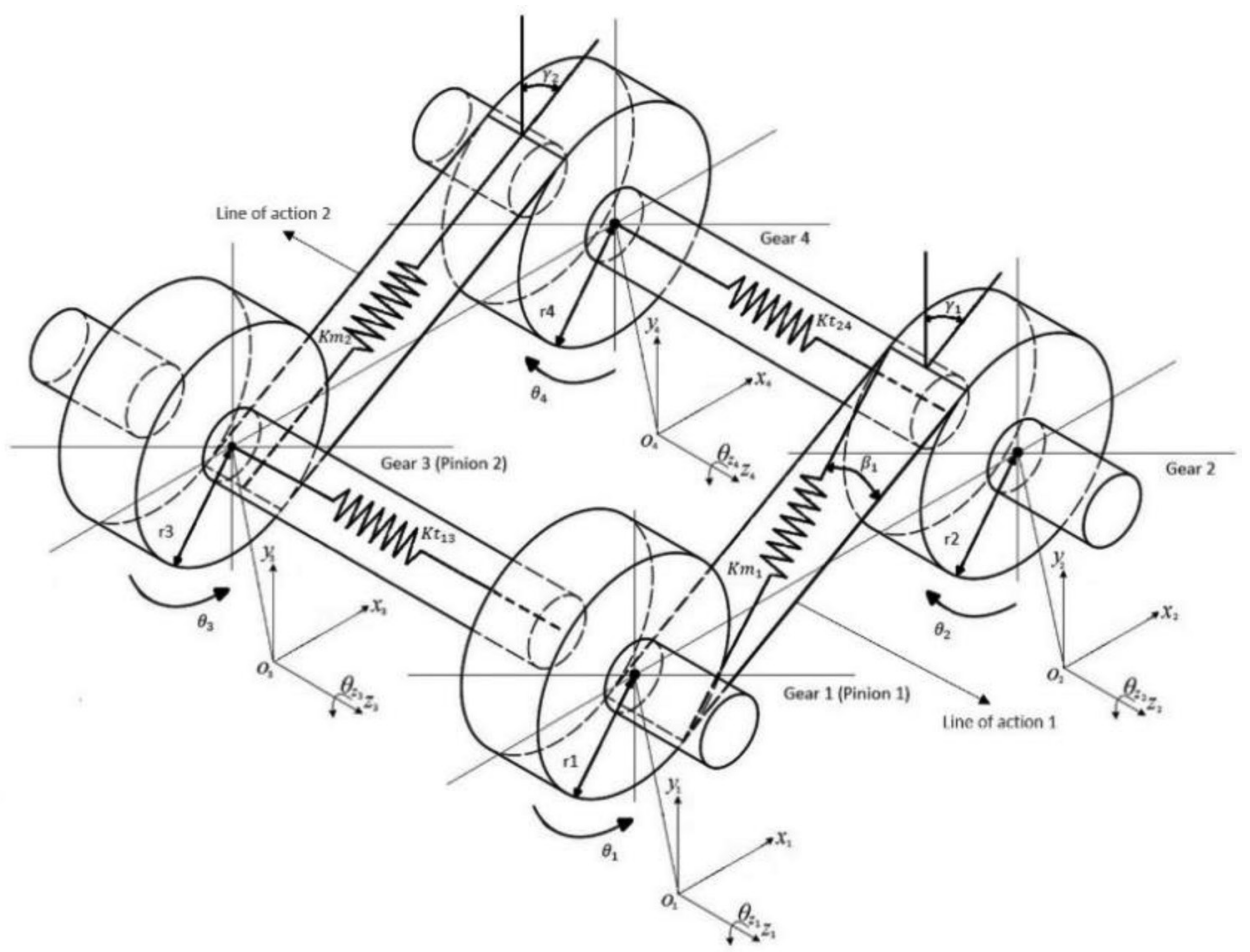

Figure 2: Lumped parameter model of the fixed-axis two-stage test rig. 
$m=9-10, n=11-12) . r_{i}$ is the circumference radius of the gears, where $r_{i}=d / 2(i=1-4)$. $K_{m 1}$ and $K_{m 2}$ are the meshing stiffness of gear pairs of stage 1 and 2, while $K_{t 13}$ and $K_{t 24}$ are the torsional stiffnesses of the shafts 1 and 2, respectively. The torsional stiffness of shaft 1 $\left(K_{t 13}\right)$ was increased by approximately three times the value of shaft $2\left(K_{t 24}\right)$ to simulate the presence of the hydraulic actuator. By using the LPM, the vibration differential equations for stages 1 and 2 were established by the following motion equations.

- Stage 1 - Helical Gears 1 and 2.

$$
\begin{gathered}
m_{1} \ddot{x}_{1}+k_{1} x_{1}+k_{2} x_{1}+k_{3} x_{1}+k_{4} x_{1}-I_{e 1} \sin \gamma_{1} \cos \beta_{1}=0, \\
m_{1} \ddot{y}_{1}+k_{1} y_{1}+k_{2} y_{1}+k_{3} y_{1}+k_{4} y_{1}-I_{e 1} \cos \gamma_{1} \cos \beta_{1}=0, \\
\ddot{\theta}_{1}+K t_{13}\left(\theta_{1}-\theta_{3}\right)-r_{1} I_{e 1} \cos \beta_{1}=0, \\
m_{2} \ddot{x}_{2}+k_{5} x_{2}+k_{6} x_{2}+k_{7} x_{2}+k_{8} x_{2}+I_{e 1} \sin \gamma_{1} \cos \beta_{1}=0, \\
m_{2} \ddot{y}_{2}+k_{5} y_{2}+k_{6} y_{2}+k_{7} y_{2}+k_{8} y_{2}+I_{e 1} \cos \gamma_{1} \cos \beta_{1}=0, \\
J_{2} \ddot{\theta}_{2}+K t_{24}\left(\theta_{2}-\theta_{4}\right)+I_{e 1} \cos \beta_{1}=0 .
\end{gathered}
$$

- Stage 2 - Spur Gears 3 and 4.

$$
\begin{aligned}
& m_{3} \ddot{x}_{3}+k_{9} x_{3}+k_{10} x_{3}-I_{e 2} \sin \gamma_{2} \cos \beta_{2}=0, \\
& m_{3} \ddot{y}_{3}+k_{9} y_{3}+k_{10} y_{3}-I_{e 2} \cos \gamma_{2} \cos \beta_{2}=0, \\
& J_{3} \ddot{\theta}_{3}-K_{t 13}\left(\theta_{1}-\theta_{3}\right)-r_{3} I_{e 2} \cos \beta_{2}=0, \\
& m_{4} \ddot{x}_{4}+k_{11} x_{4}+k_{12} x_{4}+I_{e 2} \sin \gamma_{2} \cos \beta_{2}=0, \\
& m_{4} \ddot{y}_{4}+k_{11} y_{4}+k_{12} y_{4}+I_{e 2} \cos \gamma_{2} \cos \beta_{2}=0, \\
& J_{4} \ddot{\theta}_{4}-K t_{24}\left(\theta_{2}-\theta_{4}\right)+r_{4} I_{e 2} \cos \beta_{2}=0,
\end{aligned}
$$


where $I_{e 1}$ and $I_{e 2}$ represent the internal excitation of the helical and spur gear pairs, respectively, and can be defined by the following equations:

$$
\begin{aligned}
& I_{e 1}=R_{M D 1} \cos \beta_{1}, \\
& I_{e 2}=R_{M D 2} \cos \beta_{2},
\end{aligned}
$$

where $R_{M D 1}$ and $R_{M D 2}$ are the relative mesh displacements of the helical and spur gear pairs, respectively, which can be expressed as:

$$
\begin{aligned}
& R_{M D 1}=\Delta_{K m 1} \cos \beta_{1}, \\
& R_{M D 2}=\Delta_{K m 2} \cos \beta_{2},
\end{aligned}
$$

where $\beta_{1}$ and $\beta_{2}$ are the helix angles of the pitch circle of the helical and spur gear pairs, respectively. $\Delta_{K m 1}$ and $\Delta_{K m 2}$ are the elongation and compression of the springs, representing the mating teeth along the line of action of the helical and spur gear pairs, respectively.

$$
\begin{aligned}
& \left.\Delta_{K m 1}=\left[\left(x_{2}-x_{1}\right) \sin \gamma_{1}+\left(y_{2}-y_{1}\right) \cos \gamma_{1}+r_{2} \theta_{2}-r_{1} \theta_{1}\right)\right], \\
& \left.\Delta_{K m 2}=\left[\left(x_{4}-x_{3}\right) \sin \gamma_{2}+\left(y_{4}-y_{3}\right) \cos \gamma_{2}+r_{4} \theta_{4}-r_{3} \theta_{3}\right)\right],
\end{aligned}
$$

where $x_{\mathrm{i}}$ and $y_{\mathrm{i}}$ are the translational displacement of the gears in the $x$ and $y$ directions; $\theta_{\mathrm{i}}$ is the rotational displacement in the direction of $\theta ; r_{\mathrm{i}}$ is the base circle radius of the gears $(i=1-4)$; and $\gamma_{1}$ and $\gamma_{2}$ are the normal pressure angle of the helical and spur gear pairs.

\subsection{Finite element method (FEM)}

The FEM method relied on the discretization of a generic system (continuum) into several small volumes. For each of these elements the meshing procedure assigns a set of points (depending on the type of mesh created), also called nodes, in which the solution is computed thanks to the solution of partial differential equations (PDEs) describing the equilibrium of the forces on each element. The total systems response is obtained by reconstructing results of each nodes of the structure [11]. Each component of the gear train was modelled thanks to a CAD software and simulated by an open-source FEM software to estimate the stiffnesses of the system. A three-dimensional (3-D) FE model was realized to analyse the gears (Fig. 3) and shafts (Fig. 4). The geometries of each helical gear were simplified to reduce the computation effort, while the geometries of each spur gear were entirely simulated due to its lower complexity. In addition, a two-dimensional (2-D) FE model was developed to simulate bearings (Fig. 5) to decrease their simulation complexity. The mesh stiffnesses of the stages 1 and 2 were found by simulating the mesh contact between the teeth of helical and spur gear pairs, respectively. The torsional stiffnesses of the shafts were defined by fixing the bearings position of stage 1 and rotating the corresponding gear position of stage 2 . The bearings were simulated by imposing forces on the outer rings. To compute the stiffness average values, several 

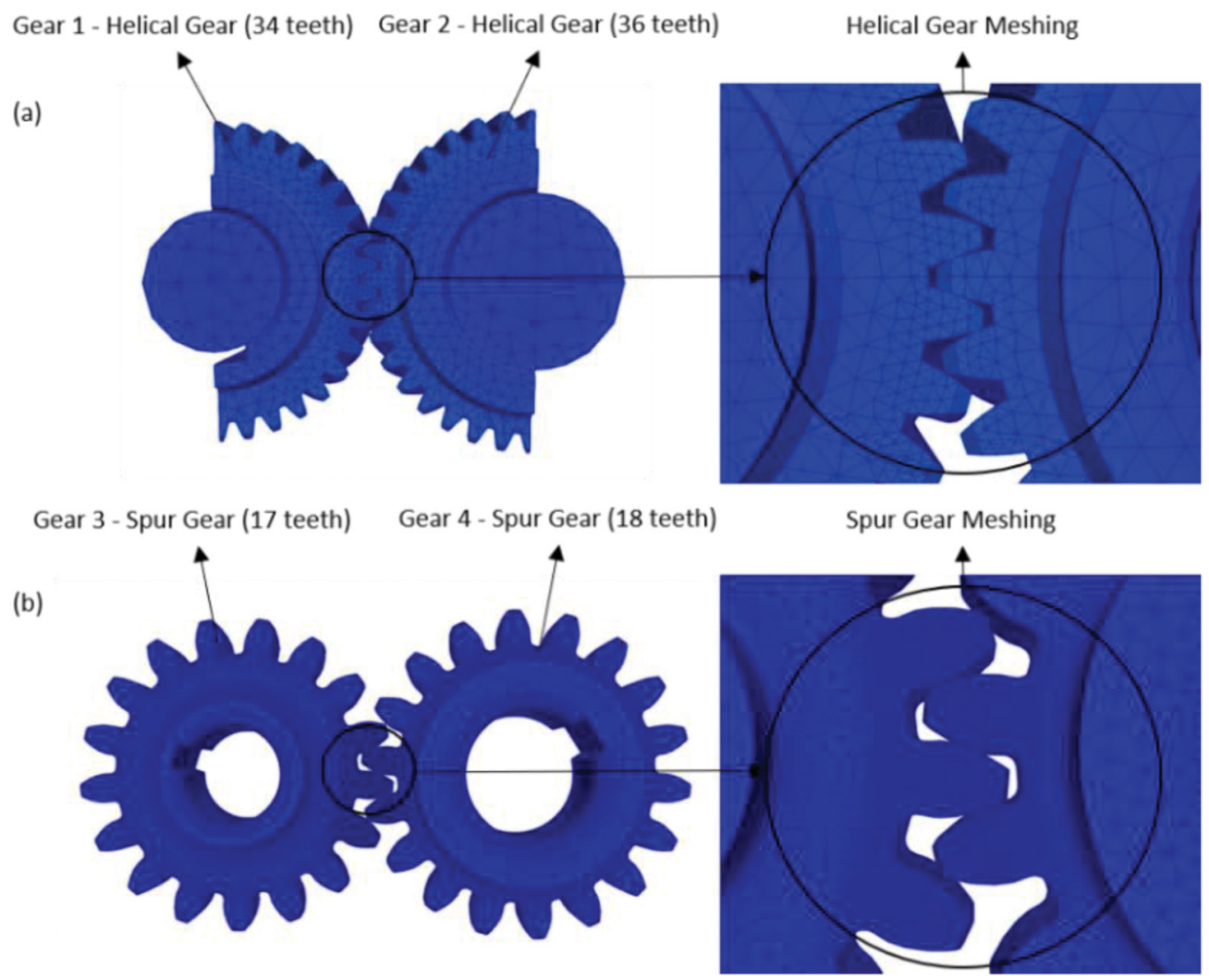

Figure 3: 3-D FE model of the (a) helical gear pair and (b) spur gear pair.

simulations in different positions were performed; 16 simulations for the helical gear pair, 17 for the spur gear pair, 17 for each shaft and 1 for each bearing type were conducted.

\subsubsection{Mesh stiffness of the spur gear pair}

In this work, the approach used to find the mesh stiffness of the spur gear pair is called "average slope approach" [12]. Using this method, the mesh stiffness is calculated as the total mesh force divided by the mesh deflection, and it can be defined combining the in-contact teeth stiffnesses [13]. The spur gear pair mesh stiffness of a single tooth contact pair can be calculated as:

$$
K_{m s 1}=\frac{1}{\frac{1}{k_{g 1}}+\frac{1}{k_{g 2}}},
$$

where $k_{g 1}$ is the tooth stiffness of the gear 1 and $k_{g 2}$ is the tooth stiffness of the gear 2 . For double tooth contact, the spur gear pair mesh stiffness $K_{m s 2}$ can be written as:

$$
K_{m s 2}=\frac{1}{\frac{1}{k_{g 1 t 1}}+\frac{1}{k_{g 2 t 1}}}+\frac{1}{\frac{1}{k_{g 1 t 2}}+\frac{1}{k_{g 2 t 2}}},
$$


where $k_{g 1 t 1}$ is the tooth stiffness of the first pair tooth (gear 1) and $k_{g 2 t 1}$ is the tooth stiffness of the first pair tooth (gear 2). $k_{g 1 t 2}$ is the tooth stiffness of the second pair tooth (gear 1) and $k_{g 2 t 2}$ is the tooth stiffness of the second pair tooth (gear 2). The components of the teeth stiffness of gears 1 and 2 are expressed as:

$$
k=\frac{F}{\delta},
$$

where $k$ is the tooth stiffness, $F$ is the normal applied force on the tooth profile and $\delta$ is the tooth deflection in the direction of the applied force. The tooth deflection can be established by the following equation:

$$
\delta=\delta_{x} \sin \gamma+\delta_{y} \cos \gamma
$$

where $\delta_{x}$ and $\delta_{y}$ are the displacements of the tooth contact element in the $x$ and $y$ direction, respectively, and $\gamma$ is the pressure angle.

\subsubsection{Mesh stiffness of the helical gear pair}

The method used to calculate the mesh stiffness of the helical gear pair is the same used to calculate the mesh stiffness of the spur gear pair. The following equations express the method used to determine the mesh stiffness of the helical gear pair for 4 (27) and 5 (28) teeth in contact.

$$
\begin{gathered}
K_{m h 4}=\frac{1}{\frac{1}{k_{g 1 t 1}}+\frac{1}{k_{g 2 t 1}}}+\frac{1}{\frac{1}{k_{g 1 t 2}}+\frac{1}{k_{g 2 t 2}}}+\frac{1}{\frac{1}{k_{g 1 t 3}}+\frac{1}{k_{g 2 t 3}}}+\frac{1}{\frac{1}{k_{g 1 t 4}}+\frac{1}{k_{g 2 t 4}}}, \\
K_{m h 5}=\frac{1}{\frac{1}{k_{g 1 t 1}}+\frac{1}{k_{g 2 t 1}}+\frac{1}{\frac{1}{k_{g 1 t 2}}+\frac{1}{k_{g 2 t 2}}}+\frac{1}{\frac{1}{k_{g 1 t 3}}+\frac{1}{k_{g 2 t 3}}}+\frac{1}{\frac{1}{k_{g 1 t 4}}+\frac{1}{k_{g 2 t 4}}}+\frac{1}{\frac{1}{k_{g 1 t 5}}+\frac{1}{k_{g 2 t 5}}},}
\end{gathered}
$$

where $k_{g 1 t 1}, k_{g 1 t 2}, k_{g 1 t 3}$ and $k_{g 1 t 4}$ are the teeth stiffnesses of 4 teeth in contact (gear 1), and $k_{g 2 t 1}$, $k_{g 2 t 2}, k_{g 2 t 3}$ and $k_{g 2 t 1}$ are the teeth stiffnesses of 4 teeth in contact (gear 2). When the helical gear pair has 5 teeth pair in contact, equation (28) is required, adding the fifth term, where $k_{g 15}$ is the tooth stiffness of the fifth pair tooth (gear 1), and $k_{g 225}$ is the tooth stiffness of the fifth pair tooth (gear 2).

\subsubsection{Torsional stiffness of the shafts 1 and 2}

The torsion along each shaft was analysed to verify the systems behaviour and to obtain the torsional stiffnesses. The linear relationship between the free end rotation angle and the applied torque can be observed in equation (29). The torsional stiffness $k_{t i}$ can be defined as:

$$
k_{t i}=\frac{T_{i}}{\theta_{i}},
$$

where $T_{i}$ is the applied torque and $\theta_{i}$ is the twist angle. Since the twist angle is required to calculate the torsional stiffness of each shaft, it can be computed thanks to the following equation. 


$$
\theta_{i}=\sin \left(\frac{\delta_{s i}}{L_{i}}\right)
$$

where $\delta_{s i}$ is the deflection due to torsion and $L_{i}$ is the distance between the load application point and the fixed end ( $i=1-2$ for shafts $1-2)$.

\subsubsection{Stiffness of the bearings}

To evaluate the stiffnesses of the bearings and study their dynamics, the FEM approach was used. Since contact forces influence the dynamic behaviour of the bearing elements $[14,15]$, also the interaction of bearings and shafts was considered. The effect of the inner and outer rings, as well as the rolling elements, were included in the simulations. To analyse the displacement of each bearing, forces were applied on the outer ring, in the $x$ and $y$ directions. Both gears 1 and 2 are supported by 4 bearings, and gears 3 and 4 are supported by 2 bearings as shown in Fig. 1. Therefore, the system [16] is composed by 4 different types of bearings, totalling 12. To analyse properly the different bearing behaviours, one simulation for each bearing type was performed (Fig. 5). The displacements found via FEM were used to estimate the stiffness of each bearing through the following equation:

$$
k_{b i}=\frac{F_{i}}{\delta_{b i}},
$$

where $F_{i}$ is the resultant force and $\delta_{b i}$ is the displacement caused by the applied force. The resultant force $F_{i}$ can be defined as:

$$
F_{i}=\sqrt{F_{i x}^{2}+F_{i y}^{2}}
$$

where $F_{i x}$ and $F_{i y}$ are the forces in the $x$ and $y$ directions.

To be used, the LPM approach requires the stiffnesses of the bearings in the $x\left(k_{b i x}\right)$ and $y\left(k_{b i y}\right)$ directions. Therefore, the stiffness of the bearings used in the LPM can be defined as:

$$
\begin{aligned}
& k_{b i x}=\frac{F_{i x}}{\delta_{b i x}}, \\
& k_{b i y}=\frac{F_{i y}}{\delta_{b i y}},
\end{aligned}
$$

where $k_{b i x}$ and $k_{b i y}$ are the stiffnesses of the bearings in the $x$ and $y$ directions $(i=1-4$ for bearings 1-4).

\section{RESULTS}

The numerical results of the FEM simulations and the criterions adopted according to literature can be found in Table 2 (helical gear pair mesh stiffness), Table 3 (spur gear pair mesh stiffness), Table 4 (torsional stiffnesses of the shafts) and Table 5 (bearing stiffnesses). 


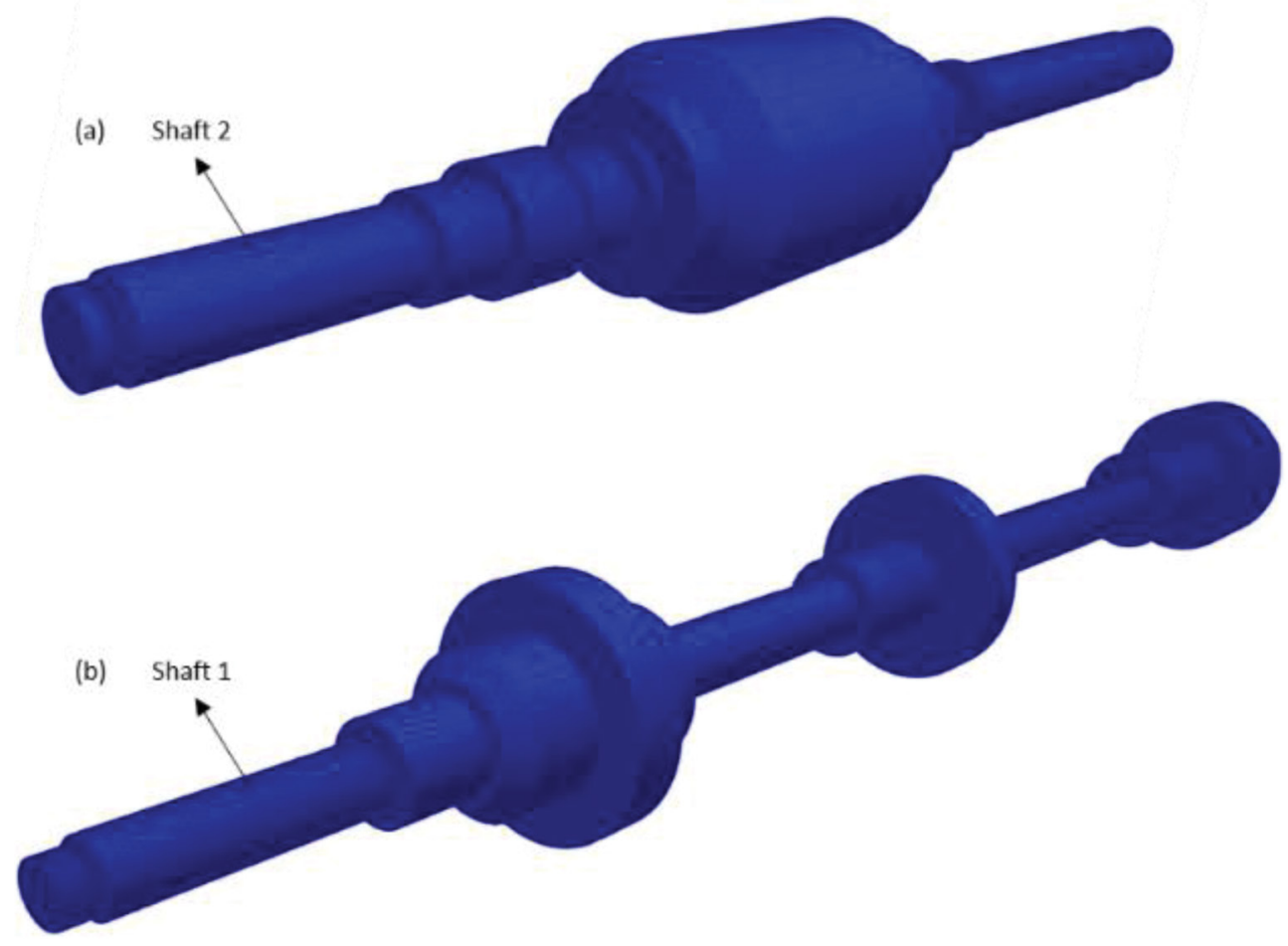

Figure 4: 3-D FE model of the (a) shaft 2 and (b) shaft 1.

Table 2: Mesh stiffness of the helical gear pair (Stage 1).

\begin{tabular}{llll}
\hline $\begin{array}{l}\text { Mesh stiffness } \\
\text { Average value }\end{array}$ & Symbol & Mesh stiffness Stage 1 & Units \\
\cline { 2 - 4 } & $K_{m 1}$ & $3,283,349.660$ & $\mathrm{~N} / \mathrm{m}$ \\
\hline
\end{tabular}

Table 3: Mesh stiffness of the spur gear pair (Stage 2).

\begin{tabular}{llll}
\hline $\begin{array}{l}\text { Mesh stiffness } \\
\text { Average value }\end{array}$ & Symbol & Mesh stiffness Stage 2 & Units \\
\cline { 2 - 4 } & $K_{m 2}$ & $1,1456,269.020$ & $\mathrm{~N} / \mathrm{m}$ \\
\hline
\end{tabular}

Table 4: Torsional stiffnesses of the shafts 1 and 2.

\begin{tabular}{llll}
\hline Torsional stiffness & Shaft $1\left(K_{\mathrm{t} 13}\right)$ & Shaft $2\left(K_{\mathrm{t} 24}\right)$ & Units \\
\cline { 2 - 4 } Average value & $2,493,959.718$ & $1,253,852.805$ & $\mathrm{Nm} / \mathrm{rad}$ \\
\hline
\end{tabular}


(a)

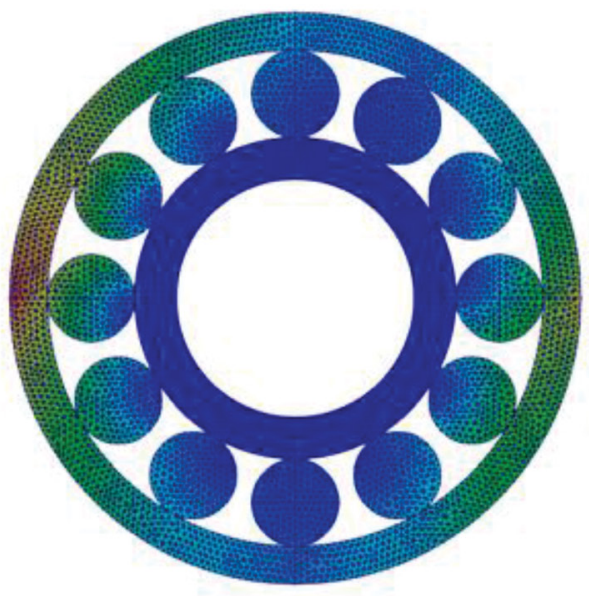

(c) Bearing 33208

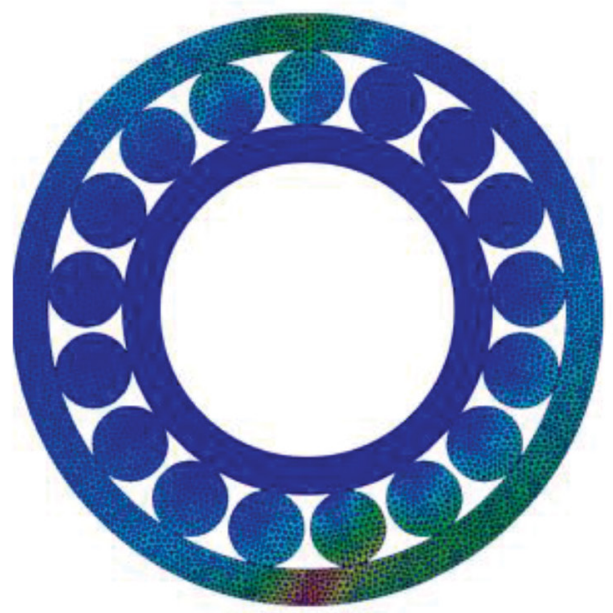

(b) Bearing NJ308

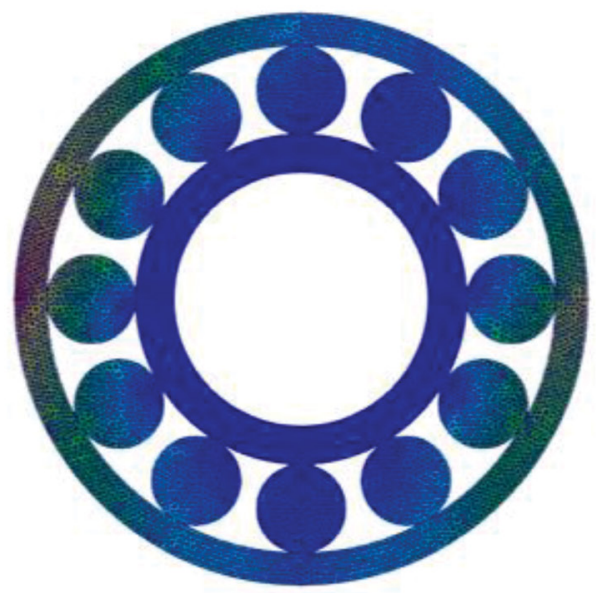

(d) Bearing 33210

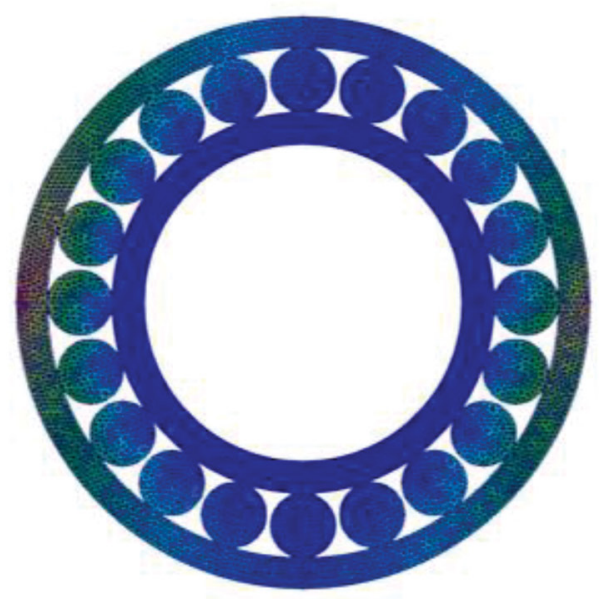

Figure 5: 3-D FE model of the bearings (a) NJ306, (b) NJ308, (c) 33208 and (d) 33210.

The responses of the gear train system were numerically computed through a MATLAB code. As output, the script returned the eigenvectors with the corresponding eigenvalues. The vibration analysis can be completed by using time domain and frequency domain techniques.

To better visualize the behaviour of the system, a fast Fourier transform (FFT) was used to convert the results from the time domain into the frequency domain.

Once all parameters of the gear train were set, the LPM was used to determine the eigenfrequencies of the entire system and to describe and analyse the vibration characteristics of the gear train. Simulations were validated with the elaboration of signals acquired during experimental tests, performed on the real system.

The vibrational behaviour of the coupled gear train system can be found in Fig. 6. It shows the comparison between the tested and simulated signals expressed in Table 6 . 
Table 5: Stiffnesses of the bearings of the (a) Stage 1 and (b) Stage 2.

\begin{tabular}{llll}
\hline \multicolumn{4}{l}{ (a) Bearing stiffnesses of stage 1 } \\
\hline Component direction & $\begin{array}{l}\text { Bearing 33208 } \\
\left(\mathrm{k}_{\mathrm{i}} ; \mathrm{i}=1-4\right)\end{array}$ & $\begin{array}{l}\text { Bearing 33210 } \\
\left(\mathrm{k}_{\mathrm{i}} ; \mathrm{i}=5-8\right)\end{array}$ & Units \\
\hline$\left(x_{i} ; i=1-2\right)$ & $787,759,199.076$ & $531,759,186.147$ & $\mathrm{~N} / \mathrm{m}$ \\
$\left(y_{i} ; i=1-2\right)$ & $827,287,972.741$ & $558,442,198.594$ & $\mathrm{~N} / \mathrm{m}$ \\
\hline (b) Bearing stiffnesses of stage 2 & & \\
\hline Component direction & Bearing NJ306 ECJ & Bearing NJ308 ECJ & Units \\
& $\left(\mathrm{k}_{\mathrm{i}} ; \mathrm{i}=9-10\right)$ & $\left(\mathrm{k}_{\mathrm{i}} ; \mathrm{i}=11-12\right)$ & \\
\hline$\left(x_{i} ; i=3-4\right)$ & $773,557,239.628$ & $332,779,262.285$ & $\mathrm{~N} / \mathrm{m}$ \\
$\left(x_{i} ; i=3-4\right)$ & $812,373,376.689$ & $349,477,710.433$ & $\mathrm{~N} / \mathrm{m}$ \\
\hline
\end{tabular}
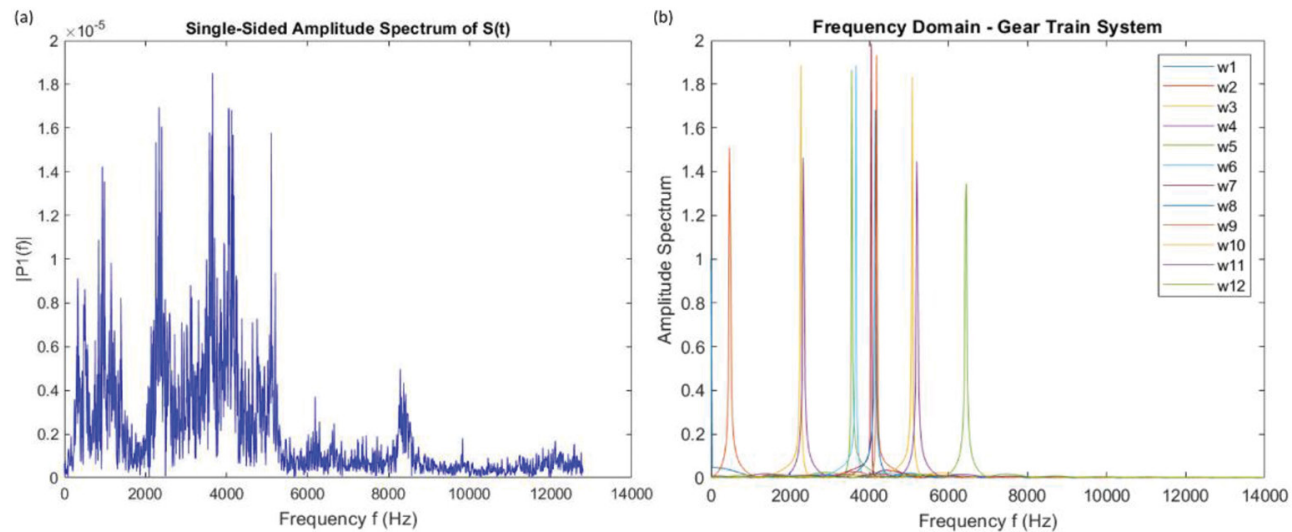

Figure 6: Charts of the (a) tested and (b) simulated eigenfrequencies of the system.

Table 6: Tested and simulated eigenfrequencies of the system.

\begin{tabular}{lllll}
\hline \multicolumn{4}{l}{ Coupled gear train system eigenfrequencies } & \\
\hline Mode & Symbol & Tested signal & Simulated signal & Units \\
\hline 1 & $\omega_{1}$ & 0.00 & 0.00 & $\mathrm{~Hz}$ \\
2 & $\omega_{2}$ & 478.07 & 475.71 & $\mathrm{~Hz}$ \\
3 & $\omega_{3}$ & $2,259.01$ & $2,262.00$ & $\mathrm{~Hz}$ \\
4 & $\omega_{4}$ & $2,334.09$ & $2,333.70$ & $\mathrm{~Hz}$ \\
5 & $\omega_{5}$ & $3,580.81$ & $3,556.90$ & $\mathrm{~Hz}$ \\
6 & $\omega_{6}$ & $3,655.80$ & $3,655.10$ & $\mathrm{~Hz}$ \\
7 & $\omega_{7}$ & $4,049.51$ & $4,041.00$ & $\mathrm{~Hz}$ \\
8 & $\omega_{8}$ & $4,143.24$ & $4,143.20$ & $\mathrm{~Hz}$ \\
9 & $\omega_{9}$ & $4,180.74$ & $4,183.50$ & $\mathrm{~Hz}$ \\
10 & $\omega_{10}$ & $5,099.38$ & $5,074.50$ & $\mathrm{~Hz}$ \\
11 & $\omega_{11}$ & $5,202.49$ & $5,202.10$ & $\mathrm{~Hz}$ \\
12 & $\omega_{12}$ & $8,286.49$ & $8,281.80$ & $\mathrm{~Hz}$ \\
\hline
\end{tabular}




\section{CONCLUSION}

Based on the analysis performed in this research, the developed FEM models seem to properly predict the stiffnesses of the components of the gear train. Masses and stiffnesses of each component can considerably affect the eigenfrequencies of the system since they are based on material and geometrical properties. By changing these parameters, it is possible to prevent unwanted vibrations that can degrade the total system. This might affect positively the operational condition of the machine if the system is unstable. The damping was neglected since the aim of this research was to determine the eigenfrequencies. The LPM results were validated with experimental data acquired from a real healthy back-to-back rig. The simulated eigenfrequencies were considered adequate and their estimation results in line with the experimental data. In conclusion, this work can be an effective starting point to setup a monitoring strategy of gearboxes and it can be a powerful tool to predict failures [17].

\section{ACKNOWLEDGEMENTS}

The authors are very grateful to the members of the Mechanical Department of the Polytechnic University of Milan for the experimental assistance.

\section{REFERENCES}

[1] Parker, R.G., Guo, Y., Eritenel, T. \& Ericson, T. M., Vibration propagation of gear dynamics in a gear-bearing-housing system using mathematical modeling and finite element analysis. Nasa Cr-2012-217664 (Issue August), 2012.

[2] Liang, X., Zuo, M. J. \& Feng, Z., Dynamic modeling of gearbox faults: a review. Mechanical Systems and Signal Processing, 98, pp., 852-876, 2018. https://doi. org/10.1016/j.ymssp.2017.05.024.

[3] Heng, A., Zhang, S., Tan, A. C. C. \& Mathew, J., Rotating machinery prognostics: state of the art, challenges and opportunities. Mechanical Systems and Signal Processing, 23(3), pp. 724-739, 2009. https://doi.org/10.1016/j.ymssp.2008.06.009.

[4] Taylor, J.I., The vibration analysis handbook. Time, 345, 2003. https://doi.org/10.1016/ bs.mie.2015.08.030

[5] Ma, X., Chen, Z., He, H., \& Zhao, Y., Research on control technologies for a highprecision multi-source vibration simulation system. Energies, 11(11), 2018. https://doi. org/10.3390/en11112956.

[6] Vullo V., Gear Volume 1: Geometric and Kinematic Design. 1st ed. Springer International Publishing, XLI 884, 2020.

[7] Liu, J.-W., Liu, J.-P., Shu, X.-B., Mikkola, A. \& Ren, G.-X., An efficient multibody dynamic model of three-dimensional meshing contacts in helical gear-shaft system and its solution. Mechanism and Machine Theory, 142, 2019. https://doi.org/10.1016/j. mechmachtheory.2019.103607.

[8] C.W. de Silva, Modeling and Control of Engineering Systems, CRC Press, 2009.

[9] Ma, H., Zeng, J., Feng, R., Pang, X., Wang, Q. \& Wen, B., Review on dynamics of cracked gear systems. Engineering Failure Analysis, 55, pp. 224-245, 2015. https://doi. org/10.1016/j.engfailanal.2015.06.004.

[10] Concli, F., Cortese, L., Vidoni, R., Nalli, F. \& Carabin, G., A mixed FEM and lumpedparameter dynamic model for evaluating the modal properties of planetary gearboxes. Journal of Mechanical Science and Technology, 32(7), pp. 3047-3056, 2018. https:// doi.org/10.1007/s12206-018-0607-9. 
[11] O.C. Zienkiewicz, Taylor, R.L. \& Zhu, J.Z., The Finite Element Method: Its Basis and Fundamentals, seventh ed., Elsevier, Oxford, 2013.

[12] Cooley, C.G., Liu, C., Dai, X. \& Parker, R.G., Gear tooth mesh stiffness: a comparison of calculation approaches. Mechanism and Machine Theory, 105, pp. 540-553, 2016. https://doi.org/10.1016/j.mechmachtheory.2016.07.021.

[13] Raghuwanshi, N. K. \& Parey, A., Effect of back-side contact on mesh stiffness of spur gear pair by finite element method. Procedia Engineering, 173, pp. 1538-1543, 2017. https://doi.org/10.1016/j.proeng.2016.12.239

[14] Cao, H., Niu, L., Xi, S. \& Chen, X., Mechanical model development of rolling bearingrotor systems: a review. Mechanical Systems and Signal Processing, 102, pp. 37-58, 2018. https://doi.org/10.1016/j.ymssp.2017.09.023.

[15] Singh, S., Köpke, U.G., Howard, C.Q. \& Petersen, D., Analyses of contact forces and vibration response for a defective rolling element bearing using an explicit dynamics finite element model. Journal of Sound and Vibration, 333(21), pp. 5356-5377, 2014. https://doi.org/10.1016/j.jsv.2014.05.011.

[16] Concli, F., Pierri, L., \& Sbarufatti, C., A model-based SHM strategy for gears development of a hybrid FEM-analytical approach to investigate the effects of surface fatigue on the vibrational spectra of a back-to-back test rig. Applied Science 2021, 11, pp. 2026, 2021. https://doi.org/10.3390/app11052026.

[17] Concli, F. \& Kolios, A., Preliminary evaluation of the influence of surface and tooth root damage on the stress and strain state of a planetary gearbox: an innovative hybrid numerical-analytical approach for further development of structural health monitoring models. Computation, 9, pp. 38, 2021. https://doi.org/10.3390/computation9030038. 